\title{
Research on the Cross Influence of Energy Consumption Structure and Industrial Structure from Perspective of Efficiency
}

\author{
Mingxiang $\mathrm{Lu}^{1, \mathrm{a}}$, Heming Xie ${ }^{1, \mathrm{~b}}$ and Guangcai Zhu ${ }^{1, \mathrm{c}}$ \\ ${ }^{1}$ School of Management, Xihua University, Chengdu 610039, China \\ alumingxiang@126.com, bxhmlai@sina.com, gh04gh@sina.com
}

\begin{abstract}
Keywords: Perspective of Efficiency; Dual Structure; Data Envelopment Analysis; Cross-effect Index ; Cross-effect Change Index

Abstract. Energy consumption structure and industrial structure are important factors affecting energy efficiency. China's energy consumption structure and industrial structure have not achieved positive interaction, but are mutual obstruction, non-coordination and low synergy. This paper studies the theory of cross influence between the energy consumption structure and the industrial structure from the perspective of efficiency. It uses Data Envelopment Analysis Method to explore the evaluation ideas of input structure and output structure on the efficiency, studies the cross influence theory of dual structure on energy efficiency, and establishes the theoretical model of measuring the cross-effect index and the cross-effect change index. It provides the theoretical basis for empirical research on the coordination and optimization of the dual structure in regions of China.
\end{abstract}

\section{Introduction}

Energy is the most basic driving force for world economic growth and social development. With the continuous increase in energy demand for economic development, energy issues have become a major challenge for global economic development. Improving energy efficiency is one of the keys to solving energy problems[1]. Domestic and foreign scholars have conducted fruitful research on the factors influencing energy efficiency. Most scholars believe that structural factors have a significant impact on energy efficiency. The way improving energy efficiency is to optimize energy consumption structure and industrial structure[2]. It has strong practical significance by studying the impact of changes in industry and energy consumption structure on energy consumption per unit of production value[3].Energy consumption structure and industrial structure are important factors affecting in energy efficiency[4,5].

The literature on energy consumption structure and industrial structure adjustment in abroad is not common, because both are formed by market mechanisms in abroad. They have influence on each other, but not the main factors affecting each other. Learning from foreign studies, domestic scholars believe that energy consumption is determined by the market, and therefore more consideration is given to the influence of industrial structure on energy consumption structure[6]. But the energy consumption structure in China is determined mainly by resource endowments and government energy policies rather than pure market behavior[7].China's energy consumption structure and industrial structure(abbreviated to dual structure) have not achieved positive interaction, but are mutually impeding[2,8], non-coordination[9] and low synergy[10]. Therefore, it is very important to study the cross-impact causing by such mutual obstruction, non-coordination and low synergy. This paper will study the cross influence theory of dual structure from the perspective of efficiency.

\section{Research Method}

As for the research on the impact of China's energy consumption structure and industrial structure on energy efficiency, domestic scholars mainly use structural energy efficiency model, structural vector autoregressive model, ridge regression method, and data envelopment analysis(DEA) method. The above studies reveal the impact of structural factors on energy efficiency from the standpoint of the energy consumption structure and the industrial structure, and do not study their cross effects on 
energy efficiency from perspective of dual structure. The research findings of the above studies are different. The reasons are related to not only the research methods and research scope selected by scholars, but also the measurement indicators and the measurement methods for energy efficiency, the selected specific indicators of energy consumption structure and industrial structure.

Energy efficiency indicators in domestic studies include energy intensity (the reciprocal of energy productivity) and energy efficiency (energy technology efficiency). The British economist Farrell[11] developed the concept of "technical efficiency" based on the researches of Debreu's[12] and Koopmans[13] on the optimal utilization of resources, and decomposed the general economic efficiency into technical efficiency and configuration efficiency. Because the technical efficiency can better point out the improvement of input or output, more in line with the "Pareto efficiency" of economics, domestic and foreign scholars have begun to use technological efficiency(TE) to study energy efficiency. This paper intends to use TE to measure energy efficiency.

In foreign studies, the production frontier theory is used to measure the technical efficiency, and the relative efficiency is compared by measuring the distance between the decision unit and the production frontier. There are parametric and non-parametric methods to measure the technical efficiency according to the production frontier theory[14]. These two methods are no advantages or disadvantages in measuring efficiency[15]. The DEA method in the non-parametric method is a method used more often while measuring efficiency [16]. This paper intends to use the Data Envelopment Analysis method to measure the cross-effects of the dual structure.

\section{Evaluation Ideas}

Under Constant Returns to Scale (CRS) assumption, we consider a decision-making unit (DMU) which uses $n$ inputs to produce $m$ outputs. $x$ represents a $n \times 1$ non-negative real input vector, $x=\left(x_{1}, x_{2}, \mathrm{~L} x_{n}\right)^{T} . y$ represents a $m \times 1$ non-negative real input vector, $y=\left(y_{1}, y_{2}, \mathrm{~L} y_{m}\right)^{T}$. Under non-considering of input structure, $n$ kinds of input are converted into the same dimension and added to $X=x_{1}+x_{2}+\mathrm{L}+x_{n}$. Under non-considering of output structure, $m$ kinds of output are converted into the same dimension and added to $Y=y_{1}+y_{2}+\mathrm{L}+y_{m}$. The evaluation ideas on the impact of input structure and output structure on efficiency are below.

Input-orientated Measure. Under input-orientated measure $(i)$, the TEs of DMU under the four input-output combinations of $(X, Y),(x, Y),(X, y)$ and $(x, y)$ may be expressed by the input distance functions $T E_{i}(X, Y)=d_{i}(X, Y), T E_{i}(x, Y)=d_{i}(x, Y), T E_{i}(X, y)=d_{i}(X, y)$ and $T E_{i}(x, y)=d_{i}(x, y)$. The input distance functions can be represented by the binary form of linear programming as follows.

$$
\begin{aligned}
& d_{i}(X, Y)=\min _{\omega, \lambda} \omega, \quad d_{i}(x, Y)=\min _{\theta, \lambda} \theta, \quad d_{i}(X, y)=\min _{\vartheta, \lambda} \vartheta, \quad d_{i}(x, y)=\min _{\delta, \lambda} \delta, \\
& \text { st } \quad-Y_{i}+Y \lambda \geq 0, \quad \text { st } \quad-\mathrm{Y}_{\mathrm{i}}+\mathrm{Y} \lambda \geq 0, \quad \text { st } \quad-\mathrm{y}_{\mathrm{i}}+\mathrm{y} \lambda \geq 0, \quad \text { st } \quad-\mathrm{y}_{\mathrm{i}}+\mathrm{y} \lambda \geq 0 \text {, } \\
& \omega X_{i}-X \lambda \geq 0, \quad \theta \mathrm{x}_{\mathrm{i}}-\mathrm{x} \lambda \geq 0, \quad \vartheta \mathrm{X}_{\mathrm{i}}-\mathrm{X} \lambda \geq 0, \quad \delta \mathrm{x}_{\mathrm{i}}-\mathrm{x} \lambda \geq 0, \\
& \begin{array}{llll}
\lambda \geq 0 & \lambda \geq 0 & \lambda \geq 0 & \lambda \geq 0
\end{array}
\end{aligned}
$$

The TEs of above are between 0 and 1.The impact of input structure on efficiency is measured with Input Structure Efficiency(ISE). The impact of output structure on input structure efficiency is measured with O-ISE. Both may be expressed by the input distance functions as follows.

$$
I S E_{i}=\frac{T E_{i}(X, Y)}{T E_{i}(x, Y)}=\frac{d_{i}(X, Y)}{d_{i}(x, Y)} \quad O-I S E_{i}=\frac{T E_{i}(x, Y)}{T E_{i}(x, y)}=\frac{d_{i}(x, Y)}{d_{i}(x, y)}
$$

The convex hull formed in the input-output combination of $(x, y)$ envelops the data points more tightly than the convex hull of $(x, Y)$, and the convex hull of $(x, Y)$ envelops the data points more tightly than that of $(X, Y)$. Thus, TE in the input-output combination of $(x, y)$ is greater than or equal to TE in $(x, Y)$, and TE in $(x, Y)$ is greater than or equal to TE in $(X, Y)$. We can draw the conclusion of that the ISE and the O-ISE are between 0 and 1. 
Output-orientated Measure. Under output-orientated measure $(o)$, the TEs of DMU under the four input-output combinations of $(X, Y),(X, y),(x, Y)$ and $(x, y)$ may be expressed by the output distance functions $T E_{o}(X, Y)=d_{o}(X, Y), T E_{o}(X, y)=d_{o}(X, y), T E_{o}(x, Y)=d_{o}(x, Y)$ and $T E_{o}(x, y)=d_{o}(x, y)$. The output distance functions can be represented by the binary form of linear programming as follows.

$$
\begin{aligned}
& {\left[d_{o}(X, Y)\right]^{-1}=\max _{\phi, \lambda} \phi, \quad\left[d_{o}(X, y)\right]^{-1}=\max _{\varphi, \lambda} \varphi, \quad\left[d_{o}(x, Y)\right]^{-1}=\max _{\psi, \lambda} \psi, \quad\left[d_{o}(x, y)\right]^{-1}=\max _{\mu, \lambda} \mu,} \\
& \text { st } \quad \mathrm{X}_{\mathrm{i}}-\mathrm{X} \lambda \geq 0, \quad \text { st } \quad \mathrm{X}_{\mathrm{i}}-\mathrm{X} \lambda \geq 0, \quad \text { st } \quad \mathrm{x}_{\mathrm{i}}-\mathrm{x} \lambda \geq 0, \quad \text { st } \quad \mathrm{x}_{\mathrm{i}}-\mathrm{x} \lambda \geq 0 \text {, } \\
& -\phi \mathrm{Y}_{\mathrm{i}}+\mathrm{Y} \lambda \geq 0, \quad-\varphi \mathrm{y}_{\mathrm{i}}+\mathrm{y} \lambda \geq 0, \quad-\psi Y_{\mathrm{i}}+\mathrm{Y} \lambda \geq 0, \quad-\mu \mathrm{y}_{\mathrm{i}}+\mathrm{y} \lambda \geq 0, \\
& \lambda \geq 0 \quad \lambda \geq 0 \quad \lambda \geq 0 \quad \lambda \geq 0
\end{aligned}
$$

The TEs of above are between 0 and 1 . The impact of output structure on efficiency is measured with Output Structure Efficiency (OSE). The impact of input structure on output structure efficiency is measured with I-OSE. Both may be expressed by the output distance functions as follows.

$$
O S E_{o}=\frac{T E_{o}(X, Y)}{T E_{o}(X, y)}=\frac{d_{o}(X, Y)}{d_{o}(X, y)} \quad I-O S E_{o}=\frac{T E_{o}(X, y)}{T E_{o}(x, y)}=\frac{d_{o}(X, y)}{d_{o}(x, y)}
$$

We can draw the same conclusion that the OSE and the I-OSE are between 0 and 1.

Impact on Efficiency by Input Structure and Output Structure. The impact on efficiency by input structure and output structure can be measured by the Input-Output Structure Efficiency( IOSE ) and the Input-Output Structure Efficiency Change Index $\left(I O S E C^{t, t+1}\right)$.

The IOSE can be considered from input-orientated measure and output-orientated measure as follows.

$$
\operatorname{IOSE} E_{i}=\frac{T E_{i}(X, Y)}{T E_{i}(x, y)}=\frac{d_{i}(X, Y)}{d_{i}(x, y)} \quad \operatorname{IOS} E_{o}=\frac{T E_{o}(X, Y)}{T E_{o}(x, y)}=\frac{d_{o}(X, Y)}{d_{o}(x, y)}
$$

The score of $I O S E_{i}$ equals to the score of $I O S E_{o}$ score, which value is between 0 and 1.

The $I O S E C^{t, t+1}$ is the geometric mean of the Input-Output Structure Efficiency Change Index at the technical level of $t$ period $\left(I O S E C^{t}\right)$ and $t+1$ period $\left(I O S E C^{t+1}\right)$. Taking input-orientated measure as an example, the IOSEC at the technical level of $t$ period and $t+1$ period can be expressed as follows.

$$
\operatorname{IOSEC}_{i}^{t}=\frac{d_{i}^{t}\left(X^{t+1}, Y^{t+1}\right)}{d_{i}^{t}\left(x^{t+1}, y^{t+1}\right)} / \frac{d_{i}^{t}\left(X^{t}, Y^{t}\right)}{d_{i}^{t}\left(x^{t}, y^{t}\right)} \quad \operatorname{IOSEC} C_{i}^{t+1}=\frac{d_{i}^{t+1}\left(X^{t+1}, Y^{t+1}\right)}{d_{i}^{t+1}\left(x^{t+1}, y^{t+1}\right)} / \frac{d_{i}^{t+1}\left(X^{t}, Y^{t}\right)}{d_{i}^{t+1}\left(x^{t}, y^{t}\right)}
$$

The $I O S E C^{t, t+1}$ can be expressed by the formula as follow.

$$
\operatorname{IOSEC}_{i}^{t, t+1}=\left\{\operatorname{IOSEC}_{i}^{t} \times I O S E C_{i}^{t+1}\right\}^{\frac{1}{2}}=\left\{\frac{\left[\frac{d_{i}^{t}\left(X^{t+1}, Y^{t+1}\right)}{d_{i}^{t}\left(x^{t+1}, y^{t+1}\right)}\right]}{\left[\frac{d_{i}^{t}\left(X^{t}, Y^{t}\right)}{d_{i}^{t}\left(x^{t}, y^{t}\right)}\right]} \times \frac{\left[\frac{d_{i}^{t+1}\left(X^{t+1}, Y^{t+1}\right)}{d_{i}^{t+1}\left(x^{t+1}, y^{t+1}\right)}\right]}{\left[\frac{d_{i}^{t+1}\left(X^{t}, Y^{t}\right)}{d_{i}^{t+1}\left(x^{t}, y^{t}\right)}\right]}\right\}^{\frac{1}{2}}
$$

If the value of $I O S E C^{t, t+1}$ is bigger than 1, it shows that the efficiency of input-output structure has increased from period of $t$ to $t+1$. If the value is less than 1 , the efficiency of input-output structure has decreased. If the value equals to 1 , the efficiency of input-output structure has not changed.

\section{Evaluation Models}

This section researches the cross-effect of dual structure from perspective of efficiency by building the evaluation models. In the models, the energy consumption structure represents the input structure, and the industrial structure represents the output structure.

Evaluation Model of Cross-effect. We can evaluate the cross influence of dual structure from the perspective of input-orientated measure and output-orientated measure, which can be measured by the 
cross-effect index of energy consumption structure and industrial structure on the efficiency $(Z)$. It is a function of the energy consumption structure $(x)$ and the industrial structure $(y)$.

Taking input-orientated measure as an example, $Z$ can be described by the function as follow.

$$
Z=f(x, y)=\frac{d_{i}(x, Y) \times d_{i}(X, y)}{d_{i}(x, y)}
$$

The value of $Z$ is between 0 and 1 . If the value of $Z$ is closer to 1 , the dual structure is more effective. We can evaluate the effectiveness of energy consumption structure and industrial structure by the value of $Z$.

Evaluation Model of Cross-effect Change. The cross-effect index of energy consumption structure and industrial structure on the efficiency will be changed while adjusting the dual structure at different periods. This change of cross-effect index can be measured by the cross-effect change index of energy consumption structure and industrial structure on the efficiency $\left(Z C^{t, t+1}\right)$.

The $Z C^{t, t+1}$ is the geometric mean of the cross-effect index of energy consumption structure and industrial structure on the efficiency at the technical level of period of $t\left(Z C^{t}\right)$ and period of $t+1\left(Z C^{t+1}\right)$.

Taking input-orientated measure as an example, the $Z C^{t}$ and the $Z C^{t+1}$ can be expressed as follows.

$$
Z C_{i}^{t}=\frac{\left[\frac{d_{i}^{t}\left(x^{t+1}, Y^{t+1}\right) \times d_{i}^{t}\left(X^{t+1}, y^{t+1}\right)}{d_{i}^{t}\left(x^{t+1}, y^{t+1}\right)}\right]}{\left[\frac{d_{i}^{t}\left(x^{t}, Y^{t}\right) \times d_{i}^{t}\left(X^{t}, y^{t}\right)}{d_{i}^{t}\left(x^{t}, y^{t}\right)}\right]}
$$

$$
Z C_{i}^{t+1}=\frac{\left[\frac{d_{i}^{t+1}\left(x^{t+1}, Y^{t+1}\right) \times d_{i}^{t+1}\left(X^{t+1}, y^{t+1}\right)}{d_{i}^{t+1}\left(x^{t+1}, y^{t+1}\right)}\right]}{\left[\frac{d_{i}^{t+1}\left(x^{t}, Y^{t}\right) \times d_{i}^{t+1}\left(X^{t}, y^{t}\right)}{d_{i}^{t+1}\left(x^{t}, y^{t}\right)}\right]}
$$

And , the $Z C^{t, t+1}$ can be expressed by the formula as follow.

$$
Z C_{i}^{t, t+1}=\left\{Z C_{i}^{t} \times Z C_{i}^{t+1}\right\}^{\frac{1}{2}}=\left\{\frac{\left[\frac{d_{i}^{t}\left(x^{t+1}, Y^{t+1}\right) \times d_{i}^{t}\left(X^{t+1}, y^{t+1}\right)}{d_{i}^{t}\left(x^{t+1}, y^{t+1}\right)}\right]}{\left[\frac{d_{i}^{t}\left(x^{t}, Y^{t}\right) \times d_{i}^{t}\left(X^{t}, y^{t}\right)}{d_{i}^{t}\left(x^{t}, y^{t}\right)}\right]} \times \frac{\left[\frac{d_{i}^{t+1}\left(x^{t+1}, Y^{t+1}\right) \times d_{i}^{t+1}\left(X^{t+1}, y^{t+1}\right)}{d_{i}^{t+1}\left(x^{t+1}, y^{t+1}\right)}\right]}{\left[\frac{d_{i}^{t+1}\left(x^{t}, Y^{t}\right) \times d_{i}^{t+1}\left(X^{t}, y^{t}\right)}{d_{i}^{t+1}\left(x^{t}, y^{t}\right)}\right]}\right\}^{\frac{1}{2}}
$$

If the value of $Z C^{t, t+1}$ is bigger than 1, it shows that energy consumption structure and industrial structure in the DMU of period $t+1$ is more coordinated than period $t$. The dual structure is continuously optimized. If the value is less than 1 , the dual structure of period $t$ is more coordinated than period $t+1$. The change of dual structure has adversely affected on energy efficiency. If the value equals to 1 , the change of dual structure from period t to period $t+1$ has no impact on energy efficiency. We can evaluate the coordination and optimization of energy consumption structure and industrial structure from period $\mathrm{t}$ to period $\mathrm{t}+1$ by the value of $Z C^{t, t+1}$.

\section{Results Analysis}

The research does the empirical study on the cross-effect of dual structure in 27 provinces of China using the evaluation models. It draws some valuable conclusions below by the empirical research.

Results Analysis of Cross-effect in regions. We use the Cross-effect model to evaluate the effectiveness of energy consumption structure and industrial structure in 27 provinces of China. Table 1 shows the results of geometric mean of Cross-effect Change by the research periods.

From Table 1, we can draw the conclusions below.

(1)The value of cross effect of dual structure in regions all take values between zero and one which demonstrates the conclusion about Formula (8). If the value is closer to 1, the dual structure is more effective. It provides the basis for evaluating the effectiveness of dual structure.

(2)The geometric mean of cross effect is different in 27 provinces. The values of Jiangxi, Fujian, Guangdong, Zhejiang, Jiangsu are over 0.9 which shows the dual structure of these provinces are more effective. But Shanxi, Qinghai, Guizhou, Gansu, Xinjiang, Inner Mongolia, Liaoning, Hebei are 
lower than 0.5 which shows the dual structure of these provinces are less effective. We should take measures to optimize the dual structure according to the scores of these provinces.

(3) The geometric mean of Cross-effect is different in four regions. The eastern region is highest, but the western region is lowest. We should pay more attention to the western region especially.

Table 1 The geometric mean of Cross-effect index and the rank in regions of China

\begin{tabular}{lcc|lcc|lcc}
\hline \multicolumn{1}{c}{ Region } & Mean & Sorting & Region & Mean & Sorting & Region & Mean & Sorting \\
\hline Beijing & 0.798 & 10 & Anhui & 0.763 & 12 & Yunnan & 0.627 & 15 \\
Tianjin & 0.555 & 18 & Fujian & 0.969 & 2 & Shaanxi & 0.562 & 17 \\
Hebei & 0.429 & 20 & Jiangxi & 0.971 & 1 & Gansu & 0.370 & 24 \\
Shanxi & 0.227 & 27 & Shandong & 0.702 & 13 & Qinghai & 0.324 & 26 \\
Inner Mongolia & 0.391 & 22 & Henan & 0.803 & 9 & Xinjiang & 0.376 & 23 \\
Liaoning & 0.416 & 21 & Hubei & 0.584 & 16 & & & \\
Jilin & 0.536 & 19 & Hunan & 0.822 & 7 & Average & $\mathbf{0 . 6 0 4}$ & \\
Heilongjiang & 0.632 & 14 & Guangdong & 0.962 & 3 & Eastern & $\mathbf{0 . 7 6 3}$ & $\mathbf{1}$ \\
Shanghai & 0.785 & 11 & Guangxi & 0.841 & 6 & Central & $\mathbf{0 . 6 3 4}$ & $\mathbf{2}$ \\
Jiangsu & 0.945 & 5 & Sichuan & 0.820 & 8 & Western & $\mathbf{0 . 4 8 6}$ & $\mathbf{4}$ \\
Zhejiang & 0.954 & 4 & Guizhou & 0.351 & 25 & Northeastern & $\mathbf{0 . 5 2 0}$ & $\mathbf{3}$ \\
\hline
\end{tabular}

Results Analysis of Cross-effect Change in regions. We use the Cross-effect Change model to measure the cross-effect change index in regions. Table 2 shows the results of geometric mean of Cross-effect Change index in the research periods.

Table 2 The geometric mean of Cross-effect Change index in regions of China

\begin{tabular}{lc|lc|lc|ll}
\hline \multicolumn{1}{c}{ Region } & Mean & Region & Mean & Region & Mean & \multicolumn{1}{c}{ Region } & Mean \\
\hline Beijing & 1.127 & Shanghai & 1.060 & Hubei & 1.062 & Gansu & 1.070 \\
Tianjin & 1.061 & Jiangsu & 1.034 & Hunan & 1.039 & Qinghai & 1.032 \\
Hebei & 1.021 & Zhejiang & 1.061 & Guangdong & 1.059 & Xinjiang & 1.050 \\
Shanxi & 1.096 & Anhui & 1.090 & Guangxi & 1.068 & Average & 1.052 \\
Inner Mongolia & 0.998 & Fujian & 1.038 & Sichuan & 1.019 & Eastern & 1.053 \\
Liaoning & 1.050 & Jiangxi & 1.033 & Guizhou & 1.062 & Central & 1.056 \\
Jilin & 1.062 & Shandong & 1.017 & Yunnan & 1.051 & Western & 1.043 \\
Heilongjiang & 1.083 & Henan & 1.020 & Shaanxi & 1.038 & Northeastern & 1.065 \\
\hline
\end{tabular}

We can draw the conclusions below.

(1)The cross-effect change index in regions at different periods are different. If the value is bigger than 1 , it shows that energy consumption structure and industrial structure is optimized from period $t$ to period $t+1$. If the value is less than 1 , the change of dual structure has adversely affected on efficiency. We should pay more attention to coordination and optimization the dual structure in those regions which the value is less than 1.

(2)The geometric mean of Cross-effect Change index in table 2 are bigger than 1 except Inner Mongolia, which shows that energy consumption structure and industrial structure is continuously optimized in the research periods on the whole. We should pay more attention to Inner Mongolia.

\section{Conclusion}

This paper studies the cross influence between energy consumption structure and industrial structure from the perspective of efficiency. It establishes the evaluation models based on the evaluation ideas to measure the cross-effect index $(Z)$ and the cross-effect change index $\left(Z C^{t, t+1}\right)$. According to the value of $Z$ and $Z C^{t, t+1}$, we can evaluate the effectiveness of energy consumption structure and industrial structure and evaluate the coordination and optimization of energy consumption structure and industrial structure from period $t$ to period $t+1$. It provides theoretical basis for empirical research on the coordination and optimization of dual structures in regions of China. 


\section{Acknowledgements}

This work was financially supported by the soft science research project of Sichuan science and technology agency(2017ZR0029), the "thirteenth five-year" plan project of social sciences of Sichuan province(SC16TJ025), and the key scientific research fund of Xihua University (Z1221508).

\section{References}

[1]Wei Chu. Research on energy efficiency of China. Zhejiang University (2009).

[2]Xiaozhe Guo, Jiali Ge. Analysis of New Indexes of Energy Utilization Efficiency Based on Dual Structure. Journal of Harbin Institute of Technology(2006), p. 999-1002.

[3]Weidong Song, Qiankun Wang. Analysis of Influence of Industrial and Energy Consumption Structure Adjustment on Energy Consumption of Production Value per Unit. China Energy(2013), p.34-37 .

[4]Tao Zhao, Honggong Shi. Research on Impact and Contribution of Energy Structure and Industrial Structure to Energy Intensity. Arid Zone Resources and Environment(2013), p.1-6.

[5]Lina $\mathrm{Xu}$, Tao Zhao, Guangwei Liu, Jinshuai Sun. Analysis on the Dynamic Effect of Energy Intensity Change and Energy Structure and Industrial Structure in China. Exploration of Economic Issues(2013), p.40-44.

[6]Dan Shi. Impact of Changes in Industrial Structure on Energy Consumption Demand. Quantitative Economic and Technical Economic Research(1999), p.50-52.

[7]Yusupu. Gulinaer. Research on the Relationship between Energy Consumption Structure and Industrial Structure. Search(2008), p.27-29.

[8]Ye Tao, Huifeng Xue. Research on the Coordination Evolution Law of Energy Structure and Industrial Structure in China. Environmental Science(2009), p.102-104.

[9]Xiaoying Wang, Jinhua Cheng, Xinhua Yi. Analysis of Coordination and Countermeasures between Industrial Structure and Energy Consumption Structure. Journal of Wuhan University of Technology (Social Science Edition) (2013), p. 201-208.

[10]Xiaoying Wang, Lei Shen, Jinhua Cheng, Yunong Lin. Coordinated Measurement Analysis of China's Industrial Structure and Energy Consumption Structure. Statistics and Decision(2016), p. 113-117.

[11]Farrell M J. The Measurement of Productive Efficiency, Journal of the Royal Statistical Society (1957), p.253-290.

[12]Debreu G. The Coefficient of Resource Utilisation. Econometrical(1951), p.273-292.

[13]Koopmans Ed. Activity Analysis of Production and Allocation(Cowles Commission for Research in Economics, New York 1951).

[14]Coelli, T.J., Rao, D.S.P., O'Donnell, C.J., Battese, G.E.. An introduction to efficiency and productivity analysis (2nd) (Kluwer Academic Publishers, Boston 2005).

[15]Peng Kang. Comparative Analysis of Parametric Method and Nonparametric Method in Economic Efficiency Research. Economy Forum(2005), p.139-140.

[16]Berger A N, Mester L J. Inside the Black Box: What Explains Differences in the Efficiencies of Financial Institutions. Journal of Banking and Finance(1997), p. 895-947. 